\title{
Dynamics of contour motion of belt drive by means of nonlinear rod approach
}

\author{
Alexander K. Belyaev, Vladimir V. Eliseev ${ }^{\dagger}$, Hans Irschik, and Evgenii A. Oborin
}

\begin{abstract}
The contour motion of the belt drive, i. e. the motion with the constant trajectory is addressed. The belt is considered as a closed Cosserat line whose particles have translational and rotational degrees of freedom. The problem is considered in the framework of geometrically nonlinear formulation with no restrictions on the smallness of displacements and rotations. The spatial (Eulerian) coordinate which is the arc coordinate in the actual configuration is introduced. The belt is divided into four segments: two contact segments on the pulleys and two free spans. The friction forces are assumed to obey the Coulomb law. The study is limited to the stationary case with the constant angular velocities of the pulleys and the equations in components are derived for both contact and free spans. In the contact segment two assumptions are employed to eliminate the unknown contact pressure and friction: (i) the full contact i. e. coincidence between the pulley and the belt and (ii) the stick condition i. e. the belt velocity is related to the pulley angular velocity. A nondimensional coordinate is introduced in the segments to obtain the boundary value problem with fixed boundaries. The boundary coordinates of the contact zones are the integration constants of the derived problem along with the other constants.
\end{abstract}

\section{Introduction}

Friction belt drives were studied extensively as they are interesting from a mechanical perspective. Until recently, one-dimensional models of extensible strings were

Alexander K. Belyaev

Institute for Problems in Mechanical Engineering, Russian Academy of Sciences, St. Petersburg, Russia

Vladimir Eliseev

Deceased on October 14, 2017, Peter the Great St. Petersburg Polytechnic University, Russia

Hans Irschik · Evgenii A. Oborin

Johannes Kepler University Linz, Austria, e-mail: evgenii.oborin@jku.at 
widely used $[13,15]$. However it turned out that the string model captures just a part of important effects in belt mechanics. Friction forces transmit power between the belt and the pulley. They are applied on the belt from one side and result not only in tangent forces, but also in distributed moments. The model without bending stiffness cannot describe the effects related to the moment loading. For this reason we apply the rod model accounting for the bending stiffness. In contact problems of the rod theory, the account of shear is known to be of crucial importance, cf. [2, 8]. The introduction of shear deformation causes the absence of lumped contact forces and promotes better understanding of the contact force distribution [3, 4]. Shear is also required to describe the effect of elastic microslip [5].

The goal of this study is to present a rational model of the belt as a rod with bending, extension and shear in the steady dynamic problem, see the results of other authors on rod steady dynamics in $[9,11]$ which are obtained without account for shear.

\section{Basic equations}

We consider the motion of a drive belt on two pulleys rotating with the angular velocities $\Omega_{1}, \Omega_{2}$ (see Fig. 1). $\kappa_{1}^{-1}, \kappa_{2}^{-1}$ are the pulley radii; they are inverse to curvature. In the initial undeformed state the belt is a circle of radius $\kappa_{0}^{-1}$. Before fitting the belt on the pulleys $\Delta_{0}=2 \kappa_{0}^{-1}-\kappa_{1}^{-1}-\kappa_{2}^{-1}$ is the center distance - the pulleys are just tangent to the belt. Then the distance increases up to the value $\Delta>\Delta_{0}$, and the belt deforms and extends forming the contact segments on the pulleys; the stress state with forces and bending moments in the belt and the contact pressure on the pulleys arises [3, 4].

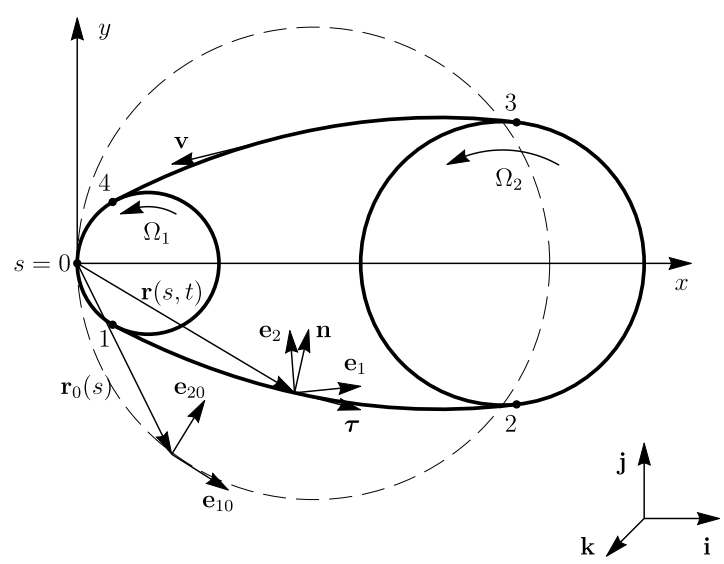

Fig. 1 Belt on pulleys 
It is reasonable to formulate the corresponding model of nonlinear elastic rod as Cosserat material line $[1,7]$. We introduce the material coordinate $0<s<2 \pi / \kappa_{0}$ in the rod; it is the arc coordinate of the belt axis in the initial state (see Fig. 1). The position vector of a rod particle is a function of coordinate and time $\mathbf{r}(s, t)$. In the initial state

$$
\mathbf{r}_{0}(s)=\kappa_{0}^{-1}\left[\mathbf{i}\left(1-\cos \kappa_{0} s\right)-\mathbf{j} \sin \kappa_{0} s\right]
$$

where $\mathbf{i}, \mathbf{j}$ are the unit vectors of Cartesian axes $x, y$ (zero refers to the values in the initial state). We differentiate and obtain the tangent and normal unit vectors

$$
\mathbf{r}_{0}^{\prime}=\mathbf{i} \sin \kappa_{0} s-\mathbf{j} \cos \kappa_{0} s=\boldsymbol{\tau}_{0}=\mathbf{e}_{10}, \quad \kappa_{0}^{-1} \boldsymbol{\tau}_{0}^{\prime}=\mathbf{i} \cos \kappa_{0} s+\mathbf{j} \sin \kappa_{0} s=\mathbf{n}_{0}=\mathbf{e}_{20} .
$$

Particles of the Cosserat line are elementary bodies with translational and rotational degrees of freedom. Therefore we associate the directors $\mathbf{e}_{1}, \mathbf{e}_{2}$ with every particle, see Fig. 1. Their coincidence with the tangent and normal (as in (1)) disappears after the deformation - it is a consequence of transversal shear. The angles $\varphi(s), \varphi_{0}(s)$ between the unit vectors $\mathbf{e}_{1}, \mathbf{e}_{10}$ and $x$-axis are important; their difference $\theta$ is rather more important, determines the particle rotation

$$
\begin{aligned}
& \mathbf{e}_{1}=\mathbf{i} \cos \varphi+\mathbf{j} \sin \varphi, \quad \mathbf{e}_{2}=\mathbf{k} \times \mathbf{e}_{1}=-\mathbf{i} \sin \varphi+\mathbf{j} \cos \varphi \\
& \varphi_{0}(s)=\kappa_{0} s-\pi / 2 ; \quad \theta(s)=\varphi-\varphi_{0} .
\end{aligned}
$$

The system of equations [7] of nonlinear elastic rods deforming in plane is

$$
\mathbf{Q}^{\prime}+\mathbf{q}=\rho \ddot{\mathbf{r}}, \quad M^{\prime}+\mathbf{k} \cdot \mathbf{r}^{\prime} \times \mathbf{Q}+m=0, \quad \boldsymbol{\theta}^{\prime}=A M, \quad \mathbf{r}^{\prime}=\mathbf{e}_{1}+\mathbf{B} \cdot \mathbf{Q} .
$$

Here $\mathbf{q}, m$ are the force and moment loads distributed per unit length, $\rho$ is the mass density per unit length, $A$ is the bending compliance, $\mathbf{B}$ is the compliance tensor of tension and shear. Usually

$$
\mathbf{B}=B_{1} \mathbf{e}_{1} \mathbf{e}_{1}+B_{2} \mathbf{e}_{2} \mathbf{e}_{2} \Rightarrow \mathbf{r}^{\prime}=\left(1+B_{1} Q_{1}\right) \mathbf{e}_{1}+B_{2} Q_{2} \mathbf{e}_{2} .
$$

We have two contact segments and two free spans in the belt (see the similar approach in [6]). In the free spans $\mathbf{q}=0, m=0$. In the contact segments $\mathbf{q}=-p \mathbf{n}+$ $f \tau, m=-h f / 2$, where $p \geq 0$ is the contact pressure, $f$ is the friction force, $h$ is the belt thickness. For the driver pulley (with the angular velocity $\Omega_{1}$ ) there is $f>0$ and for the driven pulley $\left(\Omega_{2}\right) f<0$. According to the Coulomb law we have $|f| \leq \mu p$ where $\mu$ is the friction coefficient; after exceeding the boundary of this inequality the slip begins.

The point about the character of contact is of importance: is it point-wise contact or distributed. In works $[3,4]$ based on the exact equations of the nonlinear theory of elastic rods, it is stated that the contact is distributed.

In the present paper we consider the simplest motion of the belt, which is the contour motion [15]. It means that the spatial configuration does not change in appearance in the course of time, the belt flows along the fixed closed curve with the position vector $\mathbf{R}(\sigma)$. We take the new coordinate $\sigma \in[0, L]$ to be the arc coordinate on this curve. The boundaries of the contact segments correspond to the 
values $\sigma_{1}, \ldots, \sigma_{4}$, see Fig. 1 ; they are unknown and are subject to the determination (in contrast to the string model, they are not determined by the position of the circles tangents). The new length of belt $L$ is also unknown. The contour motion law reads

$$
\sigma=\sigma(s, t) \Leftrightarrow s=S(\sigma, t)
$$

The inverse function $S$ is constructed for every fixed time instance.

For the velocity and acceleration we have the well-known formulae of kinematics

$$
\mathbf{v}=\dot{\mathbf{r}}(s, t)=\mathbf{R}^{\prime}(\sigma) \dot{\sigma}=v \boldsymbol{\tau}, \quad \mathbf{w}=\dot{v} \boldsymbol{\tau}+\kappa v^{2} \mathbf{n} .
$$

We use the formulae of differential geometry: $\mathbf{R}^{\prime}(\sigma)=\tau, \tau^{\prime}(\sigma)=\kappa \mathbf{n}$, where $\kappa$ is the trajectory curvature. In (6) the velocity of the belt motion $v=\dot{\sigma}=\partial_{t} \sigma(s, t)$ appears (with the reduced notation of the partial derivative).

Below we will use also the value $D=\left|\mathbf{r}^{\prime}\right|=\partial_{s} \sigma(s, t)$. From the equality of mixed derivatives the following is valid:

$$
\partial_{t} D=\partial_{s} v
$$

\section{Eulerian description of motion}

Thus far we have mostly used the Lagrangian description of motion [7]. However for the contour motion the spatial (Eulerian) description is more advantageous; it has the arguments $\sigma$ and $t$. Transition from one description to another is based on (5):

$$
\begin{aligned}
& u(s, t)=u(S(\sigma, t), t) \equiv \tilde{u}(\sigma, t)=\tilde{u}(\sigma(s, t), t), \\
& \partial_{s} u=D \partial_{\sigma} \tilde{u}, \quad D \equiv \partial_{s} \sigma=\left|\mathbf{r}^{\prime}\right|, \quad \partial_{t} u \equiv u^{\bullet}=\partial_{t} \tilde{u}+v \partial_{\sigma} \tilde{u} .
\end{aligned}
$$

We introduce the material time derivative here (a bullet point in the superscript denotes it). Then in formula (6) for the tangential acceleration we have

$$
w_{\tau}=v^{\bullet}=\partial_{t} v+v v^{\prime}=\partial_{t} v+\partial_{\sigma}\left(v^{2} / 2\right) .
$$

Besides the linear velocity $v$, the angular velocity $\omega$ appears in the equations

$$
\omega=\theta^{\bullet}=\left(\partial_{t}+v \partial_{\sigma}\right) \tilde{\theta}(\sigma, t) .
$$

Because the model with shear is considered, the linear and angular velocities are independent.

We make an additional simplifying assumption: the contour motion is stationary in the same sense as in the fluid mechanics [10]: $\mathbf{v}(\sigma, t)=\mathbf{v}(\sigma)$ etc. The full list of values depending only on $\sigma$ is: $\mathbf{R}, \mathbf{v}, \mathbf{Q}, M, \omega, \varphi, \mathbf{B}, \tau, \mathbf{n}, \mathbf{e}_{1}, \mathbf{e}_{2}, \mathbf{w}$. In particular, the values $\mathbf{r}, \theta, \mathbf{r}_{0}, \varphi_{0}, \mathbf{e}_{10}$ are absent in this list. This brings up the question about the bending deformation and the elasticity relation which can be written as follows: 


$$
\theta^{\prime}(s, t)=\varphi^{\prime}(s, t)-\varphi_{0}^{\prime}(s)=D \partial_{\sigma} \varphi-\kappa_{0}=A M .
$$

By now we have the system of ordinary differential equations (ODE) of stationary motion of the nonlinear elastic belt. Let us write it denoting the derivative with respect to $\sigma$ by prime:

$$
\begin{aligned}
& D \mathbf{Q}^{\prime}+\mathbf{q}=\rho \mathbf{w}, \quad D\left(M^{\prime}+\mathbf{n} \cdot \mathbf{Q}\right)+m=0, D \equiv \sqrt{\left(1+B_{1} Q_{1}\right)^{2}+\left(B_{2} Q_{2}\right)^{2}}, \\
& D \varphi^{\prime}-\kappa_{0}=A M, \mathbf{R}^{\prime}=\boldsymbol{\tau}=D^{-1}\left[\left(1+B_{1} Q_{1}\right) \mathbf{e}_{1}+B_{2} Q_{2} \mathbf{e}_{2}\right] .
\end{aligned}
$$

However the system (12) is not full; the equations for $v, \omega$ are missing. We deduce the equations from (7) and (10):

$$
v D^{\prime}=D v^{\prime} \Rightarrow v=c D, c=\text { const; } \omega=v \varphi^{\prime}=c\left(A M+\kappa_{0}\right) .
$$

We use the first equation in the second one.

The system (12), (13) consists of six ODE for the unknowns $Q_{1}, Q_{2}, M, \varphi, x, y$ - as the system in the "usual" nonlinear theory of rods deforming in plane $[3,4,8]$. In the free spans (see Fig. 1) the distributed load is absent, and on the pulleys it is the unknown normal pressure and friction force.

\section{Contact segment}

For the case without slip, i. e. when $|f| \leq \mu p$, we assume

$$
v=\Omega_{1} \kappa_{1}^{-1}+\omega h / 2
$$

(for the left contact segment). When the limiting friction is achieved at a certain segment of belt, the tangent load becomes proportional to the normal one, as the distributed moment does. Then we need to obtain the solution again accounting for these circumstances - an iteration process is probably required. The curvature radius of the belt line is $\tilde{\kappa}^{-1}=\kappa^{-1}+h / 2$. The belt coinciding with the pulley circle have the position and tangent vectors as follows:

$$
\mathbf{R}=\tilde{\kappa}_{1}^{-1}\left[\mathbf{i}\left(1-\cos \tilde{\kappa}_{1} \sigma\right)-\mathbf{j} \sin \tilde{\kappa}_{1} \sigma\right], \quad \mathbf{R}^{\prime}=\mathbf{i} \sin \tilde{\kappa}_{1} \sigma-\mathbf{j} \cos \tilde{\kappa}_{1} \sigma .
$$

We note that different assumptions may be used for the present contact model.

Using the formulae (15) and elasticity relations (12), we write the projections of the tangent unit vector into the directions of unit vectors $\mathbf{e}_{1}$ and $\mathbf{e}_{2}$ :

$$
\mathbf{R}^{\prime} \cdot \mathbf{e}_{1}=\left(1+B_{1} Q_{1}\right) / D=-\sin \alpha, \mathbf{R}^{\prime} \cdot \mathbf{e}_{2}=B_{2} Q_{2} / D=-\cos \alpha, \alpha \equiv \varphi-\tilde{\kappa}_{1} \sigma .
$$

Then similarly to the static considerations $[3,4]$ we may express the force component $Q_{2}$ as a function of coordinate $\sigma$, angle $\varphi$ and force component $Q_{1}$ in the form: 


$$
Q_{2}=B_{2}^{-1}\left(1+B_{1} Q_{1}\right) \cot \alpha .
$$

For the second contact segment we express the transverse force in the same way:

$$
Q_{2}=B_{2}^{-1}\left(1+B_{1} Q_{1}\right) \cot \left[\varphi-\tilde{\kappa}_{2}(\sigma-\tilde{L})\right] .
$$

Here $\tilde{L}$ is the arc coordinate of the belt point lying at the rigthmost point of the second pulley. In the following we focus on the first contact segment, however in the second one the analogous equations are valid. From equations (13) and (14) we obtain

$$
M=-\frac{\kappa_{0}}{A}+\frac{2}{A h} D-\frac{2 \Omega_{1}}{A h \kappa_{1}} c^{-1} .
$$

This can be used to determine the friction force from the balance of moments (12)

$$
f=\frac{2}{h} D\left(M^{\prime}+Q_{n}\right)=\frac{4}{h^{2} A} D D^{\prime}+\frac{2}{h} D Q_{n} .
$$

Also we need the equation for $\varphi^{\prime}$ derived from (12) with the use of (19)

$$
\varphi^{\prime}=\frac{2}{h}\left(1-\frac{\Omega_{1}}{\kappa_{1}} \frac{1}{c D}\right)
$$

In the balance equations of contact segments we express $Q_{1}, Q_{2}$ in terms of the tangent $Q_{\tau}$ and normal $Q_{n}$ components:

$$
Q_{1}=-Q_{\tau} \sin \alpha+Q_{n} \cos \alpha, Q_{2}=-Q_{\tau} \cos \alpha-Q_{n} \sin \alpha .
$$

Then we rewrite the equation (17) and equation for $D$ in (12)

$$
Q_{n}=\frac{\left(B_{1}-B_{2}\right) Q_{\tau} \sin 2 \alpha / 2-\cos \alpha}{(1+\cos 2 \alpha)\left(B_{1}-B_{2}\right) / 2+B_{2}}, \quad D=-\frac{1}{\sin \alpha}-B_{1} Q_{\tau}+B_{1} Q_{n} \cot \alpha
$$

The acceleration components are

$$
w_{\tau}=\left(\frac{v^{2}}{2}\right)^{\prime}=\frac{c^{2}}{2}\left(D^{2}\right)^{\prime}=c^{2} D D^{\prime} ; \quad w_{n}=\tilde{\kappa}_{1} v^{2}=\tilde{\kappa}_{1} c^{2} D^{2} .
$$

Now we write the balance of forces (12) with the use of (20) as follows:

$$
Q_{\tau}^{\prime}=\left(\tilde{\kappa}_{1}-\frac{2}{h}\right) Q_{n}+\left(\rho c^{2}-\frac{4}{h^{2} A}\right) D^{\prime}, Q_{n}^{\prime}=-\tilde{\kappa}_{1} Q_{\tau}+D^{-1} p+\rho \tilde{\kappa}_{1} c^{2} D
$$

where we need the derivative $D^{\prime}$. Now we may transform the first equation of (25) into the normal form which is not stated here, because it is lengthy. Finally from the second equation of (25) we determine the contact pressure

$$
p=D Q_{n}^{\prime}+\tilde{\kappa}_{1} D Q_{\tau}-\rho \tilde{\kappa}_{1} c^{2} D^{2} .
$$


So, in the contact zone we have two unknown functions $\varphi$ and $Q_{\tau}$ and one constant $c$ determining all the remaining variables.

\section{Free span}

The expression for curvature $\kappa$ is cumbersome and includes the derivatives of the unknown functions under the square root. Therefore it is advisable to exclude the curvature from the system of equations. To do this we use the derivative of the tangent vector $\boldsymbol{\tau}^{\prime}$ and write the acceleration vector in the form $\mathbf{w}=w_{\tau} \tau+w_{n} \mathbf{n}=$ $w_{\tau} \boldsymbol{\tau}+w_{n}^{*} \boldsymbol{\tau}^{\prime}$. Exploiting the stationarity, we account for (9) and (13) and determine the acceleration components

$$
w_{\tau}=\frac{c^{2}}{2}\left(D^{2}\right)^{\prime}=c^{2}\left(1+B_{1} Q_{1}\right) B_{1} Q_{1}{ }^{\prime}+c^{2} B_{2}^{2} Q_{2} Q_{2}{ }^{\prime}, \quad w_{n}^{*}=v^{2}=c^{2} D^{2} .
$$

Now we rewrite the balance equations without distributed loads and the remaining equations of system (12):

$$
\begin{aligned}
& Q_{1}{ }^{\prime}=\frac{\left(1-\rho c^{2} B_{2} D\right) Q_{2}\left(\kappa_{0}+A M\right)}{D\left(1-\rho c^{2} B_{1} D\right)}, \quad Q_{2}{ }^{\prime}=\frac{\left[\rho c^{2} D\left(1+B_{1} Q_{1}\right)-Q_{1}\right]\left(\kappa_{0}+A M\right)}{D\left(1-\rho c^{2} B_{2} D\right)}, \\
& M^{\prime}=\left[\left(B_{2}-B_{1}\right) Q_{1}-1\right] Q_{2} / D, \quad x^{\prime}=\left[\left(1+B_{1} Q_{1}\right) \cos \varphi-B_{2} Q_{2} \sin \varphi\right] / D, \\
& \varphi^{\prime}=\left(\kappa_{0}+A M\right) / D, \quad y^{\prime}=\left[\left(1+B_{1} Q_{1}\right) \sin \varphi+B_{2} Q_{2} \cos \varphi\right] / D .
\end{aligned}
$$

Now we subdivide the belt into four segments, $\sigma_{4}-L \leq \sigma \leq \sigma_{1}, \sigma_{1} \leq \sigma \leq \sigma_{2}$, $\sigma_{2} \leq \sigma \leq \sigma_{3}$ and $\sigma_{3} \leq \sigma \leq \sigma_{4}$. For convenience, we eventually will introduce the new nondimensional coordinate $0 \leq \xi \leq 1$ similarly to the previous works $[3,4,8]$. The main advantage of this transformation is dealing with the boundary value problem (BVP) with known boundaries. As a result we have 16 functions: $Q_{\tau}$ and $\varphi$ in the contact segments; $Q_{1}, Q_{2}, M, \varphi, x$ and $y$ in the free segments and eight constants: $c$ from (13); four boundary arc coordinates $\sigma_{1}, \ldots, \sigma_{4}, \tilde{L}$, actual belt length $L$ and center distance $\Delta$. The boundary conditions are the conditions of continuity in force, moment, position vector and angle at the segments ends; altogether there are 24 conditions in components. The formulated BVP can be numerically solved, e. g. by the collocation method of Chebfun package in Matlab.

\section{Conclusion}

The main results of the present research are listed below:

- the drive belt is modelled as a plane nonlinear elastic rod with account for bending, tension and shear; its steady motion is generalised to the case of friction contact; 
- the spatial (Eulerian) description of motion is introduced;

- the belt is divided into four segments, two contact and two free segments; the equations for these segments are combined in a single ODE system;

- the formulated BVP is written down in the form appropriate for numerical analysis;

- the simulation results will be further used to substantiate the FEM solutions, presented in $[12,14]$.

Acknowledgements This research is carried out in the framework of joint project of the Russian Foundation for Basic Research (Grant No. 14-51-15001) and the Austrian Science Fund (FWF, Grant No. I 2093 International Project).

\section{References}

1. Antman, S.S.: Nonlinear problems of elasticity. Springer, New York (2005). DOI 10.1007/0387-27649-1

2. Antman, S.S., Schuricht, F.: The critical role of the base curve for the qualitative behavior of shearable rods. Math. Mech. Solids 8(1), 75-102 (2003). DOI 10.1177/1081286503008001766

3. Belyaev, A.K., Eliseev, V.V., Irschik, H., Oborin, E.A.: Contact of two equal rigid pulleys with a belt modelled as Cosserat nonlinear elastic rod. Acta Mechanica 228(12), 4425-4434 (2017). DOI 10.1007/s00707-017-1942-0

4. Belyaev, A.K., Eliseev, V.V., Irschik, H., Oborin, E.A.: Static contact of belt and pulleys with account for shear and gravity. Journal of Physics: Conference Series (to appear in 2018)

5. Belyaev, A.K., Eliseev, V.V., Oborin, E.A.: About one-dimensional models for describing elastic microslip in belt drive. Int. Rev. Mech. Eng. 10(5), 333-338 (2016). DOI 10.15866/ireme.v10i5.8944

6. Denoël, V., Detournay, E.: Eulerian formulation of constrained elastica. Int. J. Solids Structures 48(3-4), 625-636 (2016). DOI 10.1016/j.ijsolstr.2010.10.027

7. Eliseev, V.V.: Mechanics of deformable solid bodies. St.-Peterbg. State Polytech. Univ. Publ. House, St. Petersburg (2006)

8. Eliseev, V.V., Zinovieva, T.V.: Nonlinear-elastic strain of underwater pipeline in laying process. Comp. Cont. Mech. 5(1), 70-78 (2012). DOI 10.7242/1999-6691/2012.5.1.9

9. Kong, L., Parker, R.G.: Steady mechanics of belt-pulley systems. J. Appl. Mech. 72(1), 25-34 (2005). DOI 10.1115/1.1827251

10. Loitsianskii, L.G.: Mechanics of liquids and gases. Begell House (1995)

11. Nordenholz, T.R., O'Reilly, O.M.: On steady motions of an elastic rod with application to contact problems. Int. J. Solids Structures 34(9), 1123-1143 (1997). DOI 10.1016/S00207683(96)00054-6

12. Oborin, E., Vetyukov, Y., Steinbrecher, I.: Eulerian description of non-stationary motion of an idealized belt-pulley system with dry friction. Int. J. Solids Structures (2018). DOI 10.1016/j.ijsolstr.2018.04.007

13. Rubin, M.B.: An exact solution for steady motion of an extensible belt in multipulley belt drive systems. J Mech. Des. 122(3), 311-316 (2000). DOI 10.1115/1.1288404

14. Vetyukov, Y.: Non-material finite element modelling of large vibrations of axially moving strings and beams. J. Sound Vibration 414, 299-317 (2018). DOI 10.1016/j.jsv.2017.11.010

15. Vetyukov, Y.M., Oborin, E.A., Krommer, M., Eliseev, V.V.: Transient modelling of flexible belt drive dynamics using the equations of a deformable string with discontinuities. Math. Comp. Model. Dyn. Sys. 23(1), 40-54 (2017). DOI 10.1080/13873954.2016.1232281 